\title{
Representable Model of a Finished and Unlimited 3D Cosmos
}

\author{
Marc Mignonat \\ Société d'Astronomie des Pyrénées Occidentales, Pau, France \\ Email: mmignonat@libertysurf.fr
}

How to cite this paper: Mignonat, M. (2020) Representable Model of a Finished and Unlimited 3D Cosmos. Journal of Modern Physics, 11, 976-995.

https://doi.org/10.4236/jmp.2020.117061

Received: April 28, 2020

Accepted: July 5, 2020

Published: July 8, 2020

Copyright $\odot 2020$ by author(s) and Scientific Research Publishing Inc. This work is licensed under the Creative Commons Attribution International License (CC BY 4.0).

http://creativecommons.org/licenses/by/4.0/

\begin{abstract}
A model of a 3D unlimited and finished space is presented with the philosophic prejudgement that a physical space must be representable and cannot be a virtual mathematical abstraction. This representable $3 \mathrm{D}$ space has 3 radii of curvature and so, is multiconnected as predicted by the theorem of perelman-Poincaré. This model respects the basic principles of the physics (Occam, Maupertuis, Mach, ...) and does not question much of the content of the other models. Another way of seeing is given because: 1) this model avoids the problem of the 2 infinities; 2 ) it gives an additional explanation to the expansion and to the value of the density always near the critical density; the homogeneity of the cosmos is easier to explain; 3) the attraction is always attractive and it gives an explanation to the measure of the acceleration of the expansion estimated about 6 - 7 billion years and to the great attractor; 4) it predicts the existence of many "ghosts" images, as the illusion of galaxies is older than Big Bang and these galaxies are more evolved when they are older, or for example a greater number of galaxies at a distance of about $2100 \mathrm{Mpc}$, what can be verified from the NASA/IPAC measurements of 348 galaxies of redshift $v>1 / 8 c$. Other deductions are verifiable, which should invalidate or confirm this model. In Appendix, a mathematical development deliberately simple is made to remain in a representable reality and locate any point in this space. This development can allow to make links with the spaces of Minkowski.
\end{abstract}

\section{Keywords}

Cosmology: Theory

\section{Introduction}

The purpose is to describe with curvilinear coordinates a finished and thus representable 3D space. In essence, an infinite space is not representable and from 
a philosophic point of view, a not representable entity would make us go out of the domain of the physics. Penrose [1] gives as conditions that a theory is valid if the two infinities are eliminated: the infinity of the point of origin and the outside infinity.

The opposite philosophic idea that a physical reality is not representable was remarkably developed at the atomic level by Bohr [2]. In opposition with Kant who separates the subject objector and the observed object, he considers that the object is connected to the subject. The wave or corpuscular aspects are a function of the way of observing of the subject. He is in the continuity of Lamé and Maxwell. Lamé invented curvilinear coordinates in a three-dimensional Euclidean space [3] (he assumed that the equation of Laplace is equal to zero $\Delta V=0$, and so, he described families of curves and surfaces). But we are always inside an infinite Cartesian space.

For Maxwell and his successors, the photon, described by 2 div and 2 rot with infinities, becomes an abstraction and so, is not representable.

This idea that the physics is mathematical is a principle given, among others, by Lamé, Maxwell, Bohr and recently recalled by Tegmark [4] (for him an equation creates reality). This postulate goes against the scientific method of Descartes and against the English empiricists where all start from the observation.

Before, Gauss [5] began to define coordinates on the surface of a sphere. The generalized coordinates of Gauss describe an infinite space; they allow to pass in a space with a dimension more than 2 , but, we go then into a space which becomes abstract, purely mathematical, infinite and thus non-representable. So the generalization to 3 dimensions of the formula $\cos s=\cos x \cdot \cos y$ applicable to the surface of a sphere, by considering that $s$ and $z$ are on the surface of another virtual sphere, would give a formula of type $\cos s^{\prime}=\cos x \cdot \cos y \cdot \cos z$. This formula would allow mathematical developments but does not suit us for the philosophic reason that we do not want to create a non-representable mathematical virtual world, but to stay in the physics, i.e. in a representable concrete world.

It is the philosophic position where the physics represents a reality and where the mathematics is at the service of the physics and not the opposite. Einstein and Broglie are from this current. Sommerfeld in 1923 [6], considers that "the classic undulatory theory was not replaced by a better theory and especially for the spectroscopy". For Poincaré, the mathematics is inductive and not deductive (they allow a generalization but remain not predictive) [7].

This superiority of the mathematics permeates the thought of a large number of current physicists and sometimes, unconsciously. Many cosmologists apply to the study of the cosmos, a philosophy which can be relevant but which is debatable as any philosophy. This one was established by Bohr, at the particulate level and not at the macroscopic level (we can note that it is a problem encountered by the mathematics of the general relativity (GR) when for example, we generalize Riemannian manifolds or the coefficients of Christoffel from the dimension 2 to a superior dimension). Poincaré said: "any generalization is a leap into the 
unknown." [7]

\section{Mathematical, Astronomical and Physical Aspects}

\subsection{Mathematical Aspect}

\subsubsection{General Mathematical Aspect of This Type of Space}

We take an orthonormalized space in R3 defined by 3 basic curvilinear coordinates $\boldsymbol{i}, \boldsymbol{j}, \boldsymbol{k}$. Every curvilinear coordinate $\boldsymbol{i}, \boldsymbol{j}, \boldsymbol{k}$, has a bend radius. Our goal being to present a simple astronomical model and not to make a long mathematical development, we set the same radius of curvature at the three curvilinear coordinates $\boldsymbol{i}, \boldsymbol{j}, \boldsymbol{k}$. (This condition allows to draw spherical type spaces and simply avoids spheroidal spaces in "rugby ball" more or less crushed). So, we are in a non-Euclidean space of Riemannian type (and therefore not in a space of Lobatchevsky where the radius of curvature is variable.), which allows us to respect the theorem of Lie Poincaré [7]. (This theorem imposes that in a physical space and not mathematical, it is necessary that the space be at "constant curvature" because a figure "flexible and inextensible in motion would then be deformed and therefore unable to move". "only the movement of an invariable figure is possible". This argument by Lie Poincare eliminates the hyperbolic spaces with negative curvature since in this case. The figure is distorted when it moves...).

The attachment of this radius leads to define a fourth dimension of length. (with 3 different curvature radii, a 5th and 6th dimension of length would be added).

As the curvilinear coordinates $\boldsymbol{i}, \boldsymbol{j}, \boldsymbol{k}$ are not taken as rectilinear vectors but as coordinates having a radius of curvature $r$, a point following a coordinate will describe a circle and comes back to its starting point, which has the consequence that this space defined on R3 becomes finite (Figure 1) (3 curvilinear coordinates is a sine qua non condition to have a finite space).

We can note that the same point can be located by several groups of curvilinear coordinates (The mathematical development is in Appendix).

The 2 coordinates $i, j$ will allow to define a $2 \mathrm{D}$ surface. The 3 rd curve coordinate $\boldsymbol{k}$ will define the 3D space. With 3 orthonormated coordinates $\boldsymbol{i}, \boldsymbol{j}, \boldsymbol{k}$ with the same curvature, 2 cases of figure (sphere or torus) will appear depending on whether the radii of curvature $i$ and $j$ are on the same sphere or not. (Figure 2) But, in all cases, there is a central zone that does not belong to the $3 \mathrm{D}$ space. The 3D space thus defined, is always multiconnex according to the theorem of Perelman-Poincaré which imposes that a 3D space is necessarily multiconnex.

\subsubsection{Aspect in Sphere}

It is a sphere of radius $2.414 r$ (Figure 3) with an inaccessible central "hole" and therefore not belonging to the space defined by the coordinates. This "hole" has a radius of $0.414 r$.

The curvilinear coordinate $\boldsymbol{k}$ always goes perpendicular to the surface defined by the curvilinear coordinates $i$ and $j$, which leads to a multiconnex "hypersphere" 

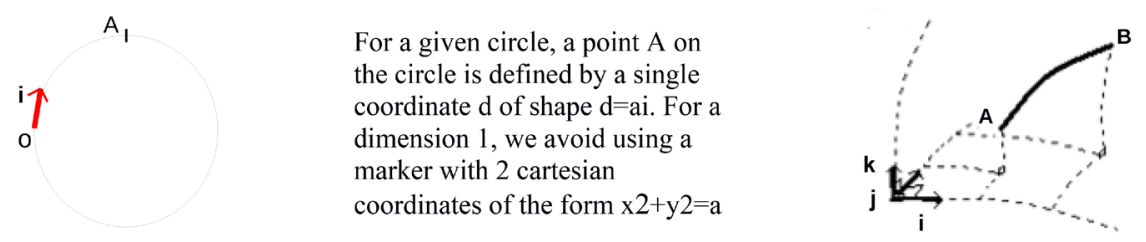

Figure 1. A 3D finite space can be defined by 3 curvilinear coordinates.

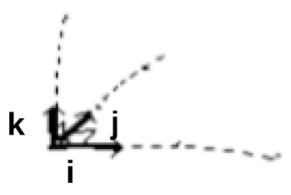

$\mathbf{i}$ and $\mathbf{j}$-are on the same sphere

Figure 2. Sphere or torus aspect.

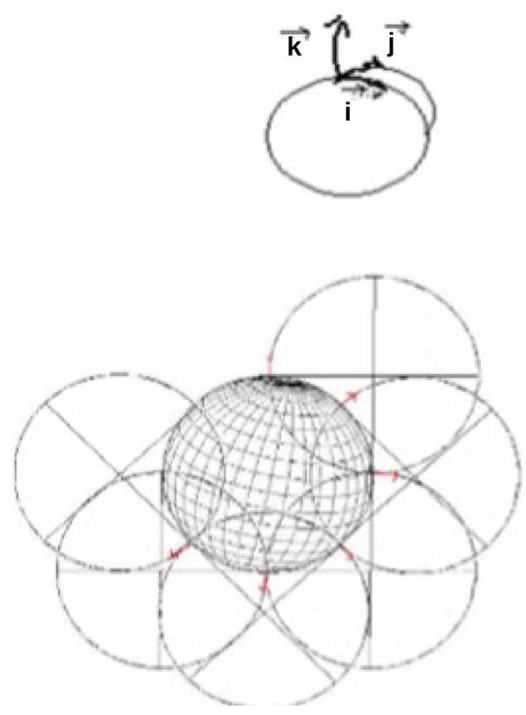

The two coordinates $\mathbf{i}, \mathbf{j}$ define a first sphere. The coordinate $\mathbf{k}$ from this sphere, creates the hypersphere

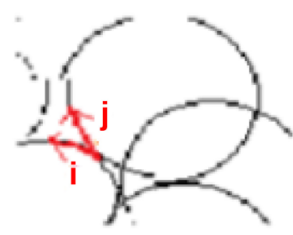

$\mathbf{i}$ and $\mathbf{j}$ are not on the same sphere (torus)

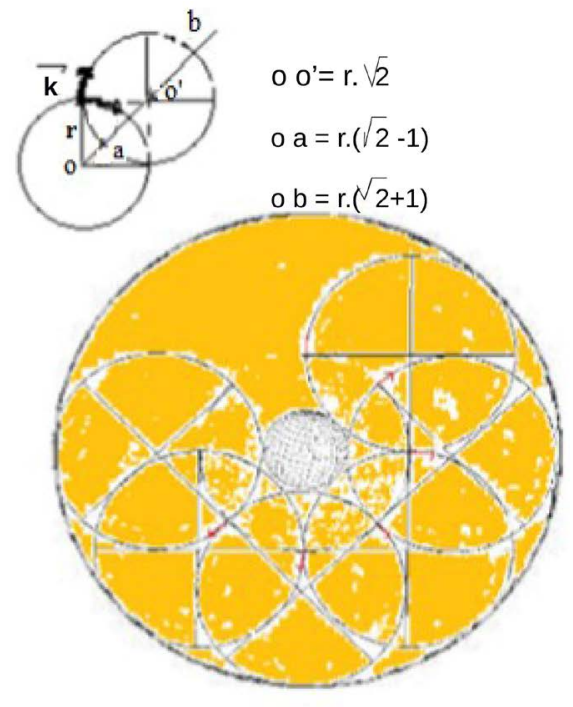

The exterior volume of the hypersphere is a sphere of radius $r(2+1)=o b$. At the centre there is a sphere of radius $\mathrm{r}(\sqrt{2}-1)=0$ a which is not accessible because it does not belong to space.

\section{Figure 3. Aspect in sphere.}

with for external boundary a sphere of radius $r\left(2^{1 / 2}+1\right)$ and for internal boundary a sphere of radius $r\left(2^{1 / 2}-1\right)$. The volume of this space is equal to the volume of the sphere of radius ob decreased by the volume of the sphere of radius oa, or, calculated from the radius $r$, Vol. $=(56 / 3) \cdot \pi r^{3}$

The surface of this space is equal to the surface of the sphere of radius ob increased from the surface of the sphere of radius oa, or calculated from the radius $r$, Surf. $=24 \cdot \pi r^{2}$.

\subsubsection{Aspect in Torus}

It is a multiconnex torus of large radius $4.414 r$ and small radius $2.414 r$ (Figure 4). 


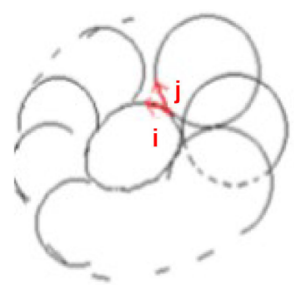

The 2 coordinates $\mathbf{i}, \mathbf{j}$ define a first torus
The coordinate $\mathbf{k}$ from the first torus, creates the hypertorus with the possibility of being in a space increased of r. $2 \sqrt{2}$ outside the circle defined according to $\mathbf{j}$ and increased by only $\mathrm{r}(2-2 \sqrt{2})$ towards the inside of this circle. This leaves a portion that is not accessible and does not belong to the space with a radius $\mathrm{r}(2 \sqrt{2}-1)$ in the centre of the circle.

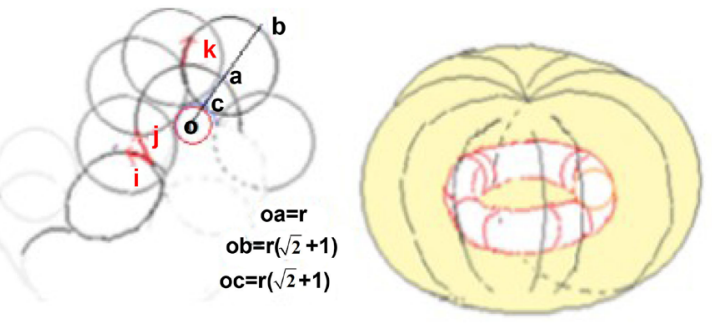

The hypertorus, multiconnex space, has a " pumpkin » aspect. It can be seen as the combination of a large external torus of small radius $\mathrm{r}(2 \sqrt{\mathbf{2}}+1)$ and large radius $2 \mathrm{r}$ and of $\mathrm{a}$ small internal torus not belonging to the space with a small radius $r(2 \sqrt{2}-1)$ and $a$ large radius $2 r$

Figure 4. Image of a 3D torus or hypertorus.

The coordinates $i$ and $j$ have their curves reversed, which creates the classical $2 \mathrm{D}$ torus.

The coordinate $\boldsymbol{k}$ goes perpendicular to the surface of this torus and thus induces multiconnex 3D hypertorus. The small radius $R_{p}$ of this hypertore will be $R_{p}=r\left(1+2^{1 / 2}\right)$ with $r=$ radius of this space. The large radius $R$ of the $2 \mathrm{D}$ torus being equal to $2 r$, the torus has the appearance "without hole" or "pumpkin". Inside the hypertorus is a torus not belonging to the space with a small radius $r\left(2^{1 / 2}\right.$ -1 ) and a large radius $R=2 r$.

The volume $V$ of the 3D torus defined from the radius of curvature $r$ is equal to $V=16 \cdot 2^{1 / 2} \cdot \pi r^{3}$

( $V_{t}=2 \pi r^{2} R$; Vol.torus $3 \mathrm{D}=$ Vol. torus with small radius $r\left(1+2^{1 / 2}\right)-$ vol.torus with small radius $r\left(2^{1 / 2}-1\right)$ with $\left.R=2 r\right)$

The surface is $S=16 \cdot 2^{1 / 2} \cdot \pi r^{2}$

$\left(S_{t}=4 \pi r R\right.$; Surf;torus 3D $=$ Surf. Torus small radius $r\left(1+2^{1 / 2}\right)+$ surf.torus small radius $\left.r\left(2^{1 / 2}-1\right)\right)$

\subsection{Astronomical Aspect: A Continuous Astronomical Space}

As in the 3D sphere (or hypersphere) of Poincaré [8] [9] or the crumpled space of Luminet [10], we find that whatever the direction in which we go, we return to the starting point. The difference is that in the space we describe, the movement can be continuous while there is a discontinuity for the spaces of Poincaré and Luminet. Indeed, Poincaré conceives "modes of conjugation of the faces of polyhedra". He associates by mathematical convention an algebraic surface that forms "cycles of edges" and "cycles of vertices" to another algebraic surface. $\mathrm{He}$ notes that these polyhedra are multiconnex. Luminet develops the dodecahedron of Poincaré to make it a model of universe and reminds [11] that "it is infinitely unlikely that the curvature of space is strictly zero..."

On a world map, in the space of Luminet, by imagining the route of a boat, arrived at the edge of the map, we make a discontinuity to extend the journey on another edge of the map. On a globe, as in our space, the motion of the ship, in 
imagination, is continuous.

The advantage of being able to include in imagination this four-dimensional space (3-dimensional length and a radius of curvature) in a Cartesian $\mathrm{R}^{3}$ orthonormated space (Cartesian space without physical reality) is that it can be represented by remaining in a 3-dimensional world.(just as the Riemanian manifolds make it possible to study a space of dimension $n$ while remaining inside this dimension) In the case of spherical aspect, each point can be located by a coordinate of type: $O S^{2}=4 R^{2}\left[\sin ^{2} w / 2(1 \pm \sin z)+\sin ^{2} z / 2(1+\sin w)\right]$, an event by a formula of type:

$O S^{2}=c^{2} t^{2}-4 R_{0}^{2}(t)\left[(1 \pm \sin z) \sin ^{2} w / 2+(1+\sin w) \sin ^{2} z / 2\right]$ with $R=R_{0}(t)$ (see details of the calculation in Appendix).

\subsection{Physical Aspect with a Spherical Representation and Curved Coordinates}

\subsubsection{Spherical Representation}

In a physical and not mathematical perspective, the principle of least energy leads to a preference for spherical representation. What we can also translate by the reminder, that isolated, a body seeks to have the maximum volume $V$ for the smallest surface $S$. In the space, a volume of liquid forms into a ball. Conversely, a red blood cell to have the largest possible gas exchange surface for a given volume will have an toroidal shape.

\subsubsection{Consequences to Have Curved Coordinates}

We can note that the 4th dimension, "the radius of curvature", if it is associated with a contraction or expansion, so with a change in length over time, could easily be associated with a temporal dimension $\left(R=R_{0}(t)\right)$ recalling the Minkowski space. (The above equation defining $O S^{2}$ is for a curved space while the Minkowski equation $\mathrm{d} s^{2}=c^{2} t^{2}-\mathrm{d} x^{2}-\mathrm{d} y^{2}-\mathrm{d} z^{2}$ is for a flat space).

In an isolated flat or curved space, If there is an expansion, the orthonormated marker stretches, the distance to be covered $(n \times(\boldsymbol{i}, \boldsymbol{j}, \boldsymbol{k})$ is always the same and the travel time does not change; However, in a curved space, the radius of curvature varies.

We are in a space with curved coordinates $R^{3}$ which is not a sub-set of a Cartesian vector space $\mathrm{R}^{3}$. This set of $\mathrm{R}^{3}$ encompasses and constitutes the cosmos (there cannot be an orthonormated space $R^{3}$ separated from a curved space $R^{3}$ ). In other words, a space cannot be both finite and infinite. If the curved space has a reality, then the Cartesian vector space is purely virtual and has no physical reality of its own since, let us remember, being infinite, by essence, it is unrepresentable; it is only a virtual mathematical tool that allows us to represent real curve space. If one considers that there is a real Cartesian space, then it makes no sense to describe a curved space and the model we present is of no interest. But to assume that there is a real Cartesian space we have to do a philosophical postulate that nothing demonstrates and for which there is no observation.

Thus, without a Cartesian reference outside the system, the travel time to 
cross the universe is the same whether the universe is contracted or very dilated, that is to say that the radius of curvature is very small or on the contrary very large. The only variable and measurable element in relation to a measurement standard is the radius of curvature which will have a lower limit (it tends towards zero when expansion grows). The variation of this radius means that there can be no strictly covalent coordinates (the enlarged image of a coordinate is not the same as the radius is different).

If, in imagination, this space with curved coordinates was included in a Cartesian vector space (reference frame outside the system), the travelled distance would not be the same: in a very contracted universe, with an external reference frame, for example, we could see the light making a few $\mathrm{cm} / \mathrm{s}$; but in the contracted curved space, these few $\mathrm{cm}$ represent the $300,000 \mathrm{~km}$ of a more dilated universe; the light still makes, in both cases $300,000 \mathrm{~km} \cdot \mathrm{s}^{-1}$ (Figure 5).

It can also be said that the ratio between the diameter of an atom and the diameter of a galaxy remains unchanged in both cases. This problem of markers is not or little addressed in the theory of expansion and when we talk about the density of the big bang at its beginning. Without an external reference to the big bang, talking about density makes no sense. To speak about density at the beginning of the big bang presupposes a frame of reference outside the system, therefore presupposes the existence of a previous space. Without an external marker, the Cartesian marker stretches with expansion and the density remains constant (Figure 6).

This problem, that we highlight, does not arise with a system with curved coordinates since the radius of curvature (which will decrease with expansion) constitutes an internal reference to the system allowing to compare volumes.

It should also be noted that there is a central $0.414 r$ radius "hole" that does not belong to space. This means that in case of maximum contraction the space cannot be reduced to a mathematical point.

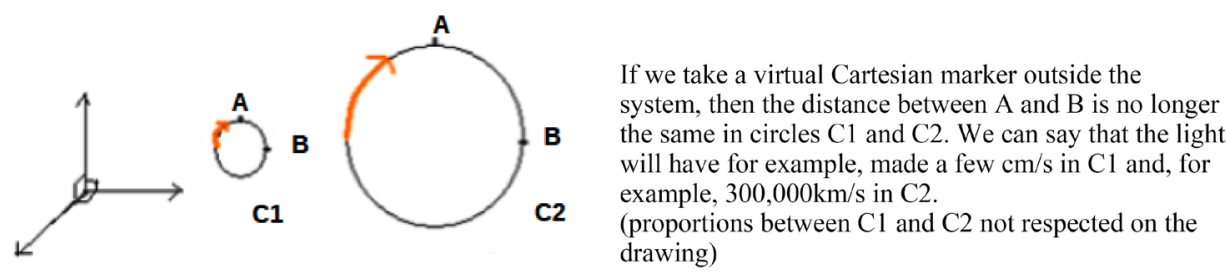

Figure 5. Cartesian marker outside the system.

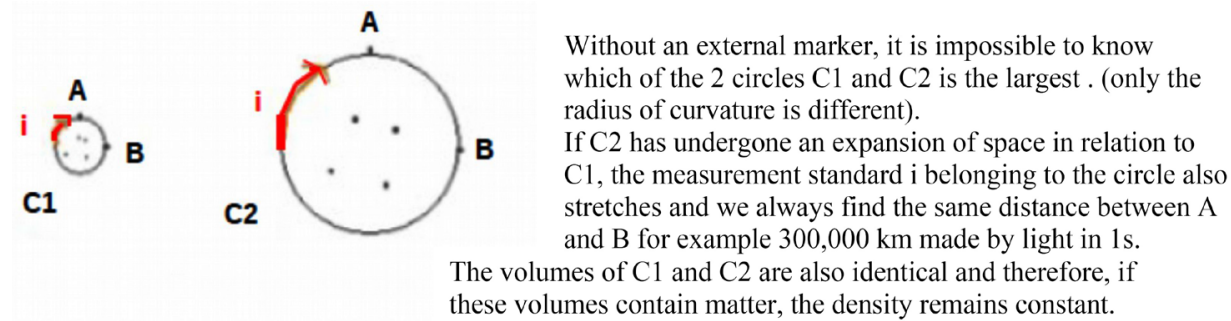

Figure 6. System without an external marker. 


\section{Cosmological Consequences or Arguments in Favor of This Finite Space}

(arguments about representation, multiple images and gravitation are to be seen as theoretical elements since they are verifiable. On GR and black holes, this is only conjectures)

\subsection{Problem of Representation}

This finite space avoids some of the problems posed by an infinite space, problems which are the same for 1, 2 or 3 dimensions.

To understand the advantage of a finite $3 \mathrm{D}$ space, it is necessary to return to the representation of the surface of the Earth which is a 2-dimensional space, finite and unlimited.

Before Anaximandre in antiquity or the arrival of the Jesuits in China, the Earth is a flat disk; a space with 2 dimensions infinite and unlimited since we can always enlarge the plateau or extend a stick beyond the plateau. The understanding of the world is then very complex and calls for insoluble contradictions comparable to our current problem of limits (How do the stars turn and how do they pass under the plateau? What is behind the plateau? Where does the water fall? Does it go into a parallel world? another dimension? a hyperspace or does it pass, as it seems today, by a wormhole to go from west to east?) In ancient Egypt, it was said that the goddess Nout swallowed the sun to the west and gave birth to it in the morning to the east. With our current language, they would have talked about hyperspace and wormhole.

On the surface of the earth, we can find ourselves in 2D; the curvature of the earth (3rd dimension) is only an intellectual construction that we do not use in the system of longitudes and latitudes. Rotondity is a concept that provides a better intellectual understanding of the Earth than a flat Earth.

Similarly, in our curved space where we can walk in $3 \mathrm{D}$; the 4 th dimension of curvature is not necessary to locate us, but this 4 th dimension is essential to build a finished space.

Of course, it is not because a concept allows for simpler representation that it proves its validity. But reject this concept for this reason is reject the representation allowed by a round Earth and continue to search for how to represent a flat Earth. Now, the simplest representation is the most likely to be true: it is the principle of simplicity (lex parcimoniae) or the principle of the Occam razor.

NB: Note on the traveler of the Middle Ages coming to the end of the world and extending his stick. In our spherical 3D space, it might be pointed out to us that the traveler can always extend his stick, but it is forgetting that here the traveler does not walk on an infinite and unlimited 2D plane but on a 2D sphere; therefore, it will remain in a finite zone and cannot go to infinity; moreover, a stick of astronomical length would bend (Figure 7).

\subsection{Multiple Images}

The 2 nd property of a finite space means that a specific event located at a distance 


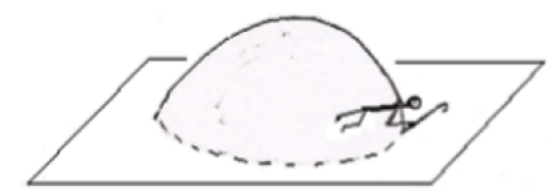

The traveler walks on an infinite plane. So he does not find the finitude of the world and can always extend a stick.

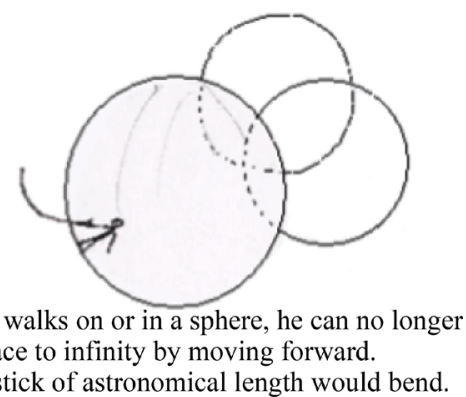
enlarge his space to infinity by moving forward.
In addition, a stick of astronomical length would bend.

Figure 7. Philosophical problem of the traveller of the middle ages.

$\mathrm{x}$ can be perceived at least once, and in an opposite direction at a different distance.

Finite spaces (circle and sphere) have properties that can also be found in 3D spaces such as the Poincaré hypersphere [8], the crumpled spaces of Luminet [10] or the space described above with curved vector markers.

1) Irrespective of the direction we take, we always go back to where we started.

2) The radius of curvature remains the same when we consider only a dimension 2 of the 3D space or a dimension 1 (a circle inscribed in a sphere has the same radius as the sphere).

\subsubsection{General Case}

In this curved space diagram, whatever the object $\mathrm{A}$, there will always be an image $\mathrm{A}^{\prime}$ "in opposition on the circle linking $\mathrm{B}$ (observation point) and $\mathrm{A}$.

The shorter the BA' distance, the greater the BA" distance; the total is $14 \times 10^{9}$ light years (assuming a perimeter equal to $14 \times 10^{9} \mathrm{LY}$ ).

An analogy can be made to sound on Earth. So let us assume that a vector such as sound can be heard throughout the Earth. Let us suppose there was a punctual explosion in London and we were located on the meridian of Greenwich at $1000 \mathrm{~km}$, for example in Tarbes. We will hear the sound in Tarbes 1 hour after the explosion, but also about 39 hours later. (We assume a straight-line path and do not assume that the sound could travel around the Earth several times.) If we do not know that we are on a sphere, but that we believe ourselves on a plane, then we will have recorded 2 explosions located in opposite directions and at distances of 1 hour and 39 hours of journey-sound.

We make here the hypothesis of not deviated light paths, which leads to a spherical geometry with 2 opposite images (Figure 8).

A verification would be possible if a galaxy was precisely identified, if we knew how it was going to evolve or if we identified particular characteristics, allowing us to say that this galaxy in another region of the sky is, actually the same galaxy seen at a different age. The idea of 2 images at different ages of the same object can also be found in a particular group or configuration of galaxies that can be more easily predicted mergers, closers, moves away, etc. In the hypothesis of this curved space, it must be considered that all objects located very far away, for example beyond 12 billion LY, are in fact very close to each other (Figure 9). 


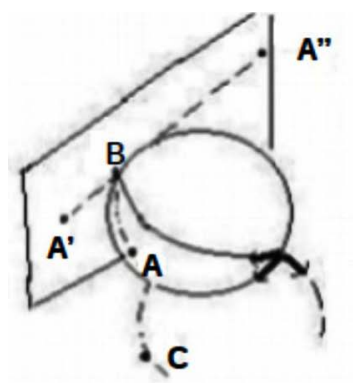

For observer B, the images of $\mathrm{A}$ appear in $\mathrm{A}^{\prime}$ and A',

$A^{\prime}$ at 4 billion LY

A' 'at 10 billion LY

A light starting from $\mathrm{C}$ can reach in $\mathrm{B}$ by following a direct path $\mathrm{CB}$ with a smaller radius of curvature.

Figure 8. General case with 2 images of the object A.

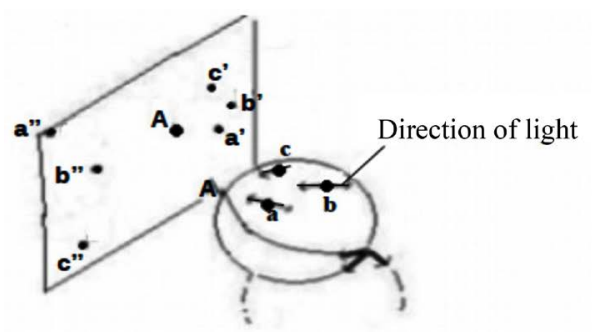

If we consider the actual position of $\mathrm{A}$ and 3 relatively nearby objects $a, b, c$ on a sphere, the observation in $\mathrm{A}$ in the plane will give the images of 3 old nearby objects a',b',c' and 3 very distant young objects a", b", c"'.

Figure 9. The 3 distant objects a", b", c" are in fact the images of the nearby objects a, b, c.

If we imagine a sphere with a perimeter of $14 \times 10^{9} \mathrm{LY}$, then there is an average deviation of $1^{\circ}$ every $40 \times 10^{6} \mathrm{LY}$.

NB: If the volume of this space is calculated from a radius $R\left(1+2^{1 / 2}\right)$, its radius remains equal to $R$.

\subsubsection{Particular Case: When A Is Located at $180^{\circ}$}

(Figure 10) in a direction according to the coordinates $i, j$ or $k$, in fact the farthest possible point, the images $A^{\prime}$ of the object $A$ will be located on a celestial circle, All of them are about $7 \times 10^{9}$ years old.

This means that for this distance, the images of a galaxy will be in greater number and a peak of galaxies should be observed at this distance. We took a survey of the 348 galaxies with a $V>1 / 8 c$, taken from the NASA/IPAC catalogue [12], which seems to confirm this hypothesis (Figure 11).

The peak of galaxies is between 2000 and $2500 \mathrm{Mpc}$ and would correspond to a half-turn (median at $2260 \mathrm{Mpc}$ when taking galaxies between 0 and $4500 \mathrm{Mpc}$ ). The complete turn would give the time since the big bang either $4500 \mathrm{Mpc}+/-$ $500 \mathrm{Mpc}$ or $14.7+/-1.6$ billion LY.

This histogram with a second peak may also suggest that the light could have gone more than one turn. But this hypothesis requires a larger statistical series to be verified; in this case, we will have the illusion of perceiving galaxies of age greater than 14 billion years with the apparent paradox that they are all the more evolved as they are old.

\subsubsection{Particular Case at $360^{\circ}$ (Figure12)}

At $4400 \mathrm{Mpc}$ it is an image of ourselves, $14 \times 10^{9}$ years ago. The image can follow all the meridians and make a complete turn to arrive. The image will be found 


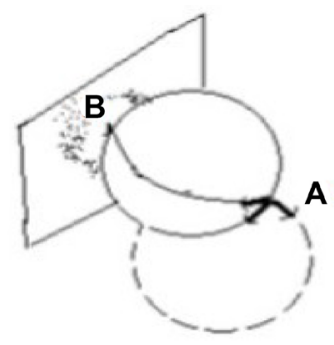

Figure 10. Objects at $180^{\circ}$.
Light can follow all paths in the sphere of diameter AB. For the B observer, the light from $A$ will form a circle of images in a plane perpendicular to the diameter $\mathrm{AB}$. The light, starting from $A$ on a path which is not on the surface of the sphere of diameter $\mathrm{AB}$, cannot reach point $\mathrm{B}$.

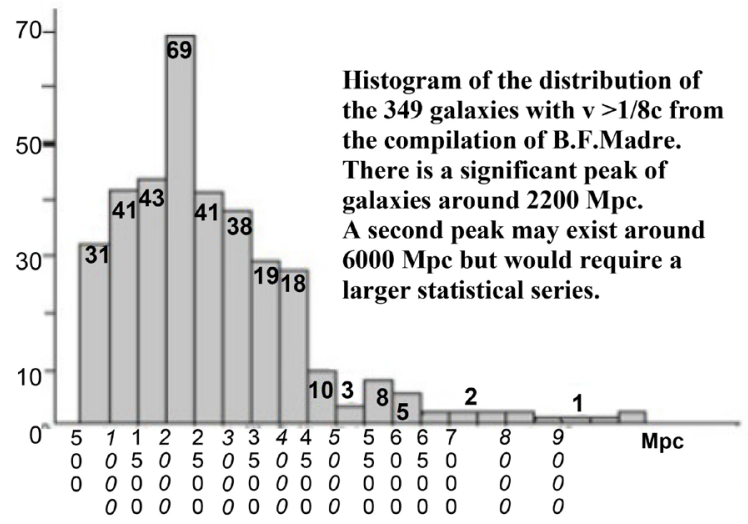

\author{
3 galaxies around \\ $14,000 \mathrm{Mpc}$ are not \\ reported. \\ 7 galaxies were isolated \\ from the results due to \\ inaccuracy in their \\ distance $(2000 \mathrm{Mpc}$ \\ difference between 2 \\ measurements)
}

Figure 11. Peak of galaxies at $180^{\circ}$.

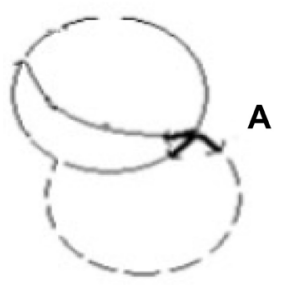

Figure 12 . Objects at $360^{\circ}$.
The light from A returns to A. All the trajectories being circular, whatever the direction taken, go back to point $\mathrm{A}$. The images from $\mathrm{A}$, for the observer in $\mathrm{A}$, will reach from every point in space and form a celestial sphere.

It is the image of the CMB, from a single point, which comes to us from all directions of space.

throughout the celestial sphere, so will be identical in all directions (for the cosmic microwave background, the differences of temperature around $3^{\circ} \mathrm{K}$ can only be an indication of the hazards encountered by light during its journey and/or images of the different faces of the same object; so, there is no problem in explaining the homogeneity of the universe since every point in the celestial sphere is the image of the same point.). But nothing extraordinary for the theory of the Big bang and it is finally easier to imagine it by recalling that it took place also where we are rather than elsewhere at $4200 \mathrm{Mpc}$, in an undetermined direction

\subsubsection{Beyond $360^{\circ}$}

There is nothing to prevent the light from doing more than one turn and therefore having the illusion of a galaxy older than the big bang with the paradox that the farther away it is, the more evolved it is. (Perhaps this is what the survey from the NED catalogue above suggests with the reservation that the series stu- 
died is too small to be significant on the second peak.)

Let us remember that in our curved universe, a large part of the universe is not visible from a given point. Because of the curvature, we cannot see beyond a diameter of $4.5 \times 10^{9} \mathrm{LY}$ in a space with a diameter of about $11 \times 10^{9} \mathrm{LY}$. To see beyond $4.5 \times 10^{9} \mathrm{LY}$, you would have to travel. If light can reach us from other points in space (Figure $7(a)$ ), there are points from which it cannot reach us (Figure 9).

\subsection{Gravitation Is Always Attractive, Does Not Need to Be Repulsive}

In this curved space, all bodies moving away from each other will, at a given moment get closer without this rapprochement meaning a contraction, the expansion (corresponding to an increase of the radius $r$ ) not being prevented from continuing. The analogy with the drift of the continents can be made: (Figure 13) we start from the Pangea, the continents drift and then, little by little, get closer. (In the hypothesis of expansion, it is the space that grows between galaxies and not galaxies that move, and we can continue the analogy by saying that it is not the continents that drift but the mantle of Earth that grows from the dorsals).

Because of the attraction that the continents exert on each other, the continents will accelerate their closeness, so as part of the expansion, we will have the impression of an acceleration of the expansion. For example, if we consider the America continent moving away from Europe; America moving closer to Asia, the growing attraction of Asia causes an acceleration of the displacement to the west. Seen from Europe, America seems to be accelerating its distance and one can falsely conclude that the attraction between Europe and America has become repulsive. The speeds of galaxies are increasing! They seem to be moving away, but they are actually getting closer! We have here a hypothesis of explanation of the paradox between expansion on the one hand, acceleration of expansion and galactic moving closer towards a point without mass: "the great attractor" on the other.

Gravitation remains attractive, it's reassuring!

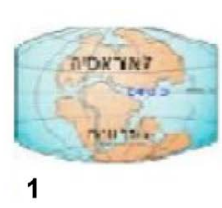

The continents move away and then move closer together without it meaning a contraction. The continents with the "impulse » of departure, past $180^{\circ}$ attract again and accelerate, converge and will cross towards the center of the Pacific at the level of the « great attractor ».

Size increase from 1 to 3 represents expansion.
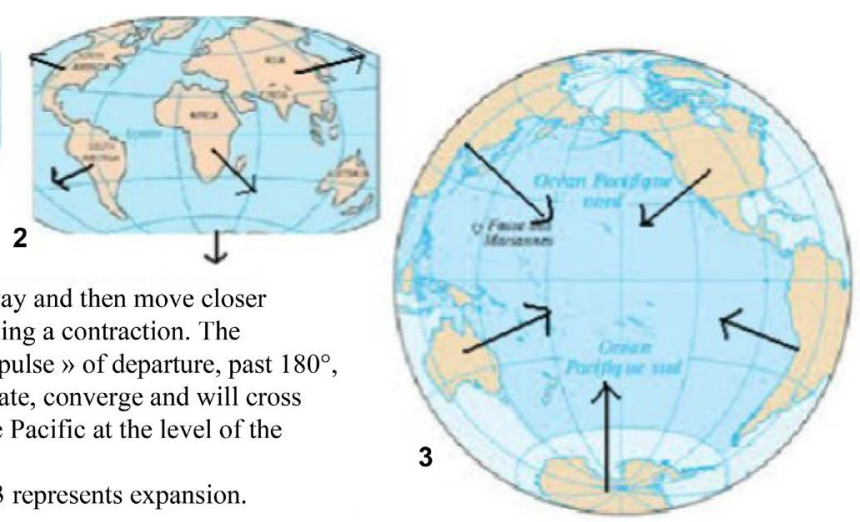

Figure 13. Explanation of acceleration of expansion by gravitation. 
Verification: always taking the hypothesis of a 3D sphere of perimeter 14 billion LY, the appearance of the acceleration will be located when $180^{\circ}$ are traversed, therefore around 7 billion AL. Galaxies will converge to a crossing point at the level of the "great attractor" and it will be necessary to distinguish the acceleration due to the attraction of the masses from the not necessarily increased acceleration of the expansion.

A verification could be seen on the representation of Laniakea [13] where galactic movements are curved and in the direction of an apparently empty point. If the displacements are actually on one or more spheres, then the galaxies face each other and tend to move closer together (see Drawing 3 of Figure 12). It is then logical to see them curved trajectories and their accelerations towards the point of convergence can be calculated in a conventional way.

\subsection{Conjecture: Does This Space Allows to Give a Representation of the 3D Space Curve of General Relativity? Does a Radius of Curvature Lead to the Existence of a Centrifugal Force?}

The surface of the Earth is a curved, finite 2D space. By applying the mathematical formalism found in general relativity (GR), an object moving on its surface cannot leave this surface, follows a geodesic and therefore does not, by definition, undergo centrifugal force. It is the introduction of the 3rd dimension (the terrestrial radius) which, mathematically, allows a displacement in a direction parallel to the radius and thus authorizes the inertial forces (an aircraft follows a geodesic and undergoes centrifugal and Coriolis forces).

The GR mathematically describes a non-representable 3-dimensional curve space. As with the previous $2 \mathrm{D}$ space, any object follows a geodesic where by definition there is no centrifugal force. In the curved space we describe, the existence of a radius of curvature (4th dimension) would lead, as in the previous case, that any object moving with a velocity $\mathrm{v}$ would undergo a centrifugal force even if it followed a geodesic. The GR is a principle, not in opposition to the Newtonian theory [14] which is neutral on the question of the finite or the infinite of space; the question of a 4th dimension of length, constitutive of space is irrelevant for the GR. It is also necessary to recall that the GR is placed in a 3-dimensional curved space, built by a generalization of a 2-dimensional space. So, it is connex, thus not belonging to the world of physics but only to that of mathematics. Now, the Poincaré conjecture become theorem of Perelman Poincaré previews that "any closed dimension 3 manifold having a finite fundamental group has a spherical geometry", that is to say is covered by the 3-sphere. This space of dimension 3 is therefore necessarily multiconnex.

Logically, the calculation of this centrifugal force, if it existed, would make it possible to know the radius of curvature of the space (Another way to measure the radius would be to follow two different paths between 2 points $A$ and $B$ and measure different gyroscopic orientations).

This hypothetical centrifugal force will be stronger when the radius is smaller and this can then be a hypothesis to the engine of the expansion of the Universe. 
So, we arrive at a balance between force of attraction and centrifugal force which would imply that we would tend towards the critical density which would in fact be a density of equilibrium.

If we want to avoid talking about centrifugal force, we can reason from entropy. Entropy $S$ is increased when the matter disperses in a larger volume $(\delta S=$ $-P d V$ ). But if, at the same time, the volume increases because of the enlargement of the reference mark linked to the expansion, $\left(R=R_{0}(t)=\right.$ comobile distances and therefore comobile volume) the measured volume is identical, the density of the matter is identical and the increase of entropy is then zero (Figure 14).

It is not forbidden in the space we describe, that areas are expanding and others locally, such as black holes, are contracting.

In this $3 \mathrm{D}$ space we can make the comparison with the surface of the earth which is a $2 \mathrm{D}$ surface but embossed between the seabed and the high mountains. Perhaps, the problem of the finiteness of our 3D space is no different from the 2D surface of the Earth. We cannot leave 2D space even if the relief deforms this space or if an expansion or contraction of the Earth takes occur.

\subsection{For Black Holes (BH)}

The multiconnex space described above is also to be studied within the black hole which is a contraction zone.

When the mass of the $\mathrm{BH}$ increases, the radius of Schwarzschild increases, the radius of the $\mathrm{BH}$ itself is supposed to decrease and it is accepted that the $\mathrm{BH}$ curves more and more the space that is here in contraction. This curvature being made in 3 dimensions, the theorem of Perelman-Poincaré imposes a multiconnex space. If we just assume that the curvature of the space is done according to my orthonormated curved marker in $\mathrm{R}^{3}$, then it will appear at the center of this hole for sufficient density, an area of radius $R\left(2^{1 / 2}-1\right)$ not belonging to this space.

This central zone not belonging to space will always exist even if the radius decreases.

This hypothesis of a 3-dimensional curved multiconnex space eliminates the problem of infinite density at one point. However, it does not eliminate the problem of unlimited increasing density but may open the door to simpler conjectures.
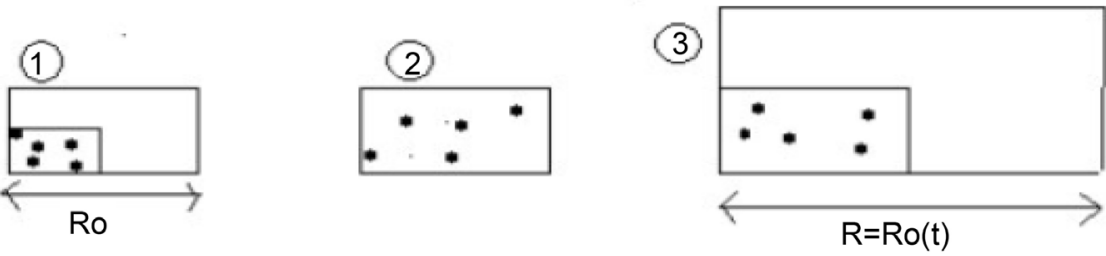

Between 1 and 2 the mass disperses in a larger volume. Entropy $\mathrm{S}$ increases. But, if at the same time with the expansion, the marker is streched, then volume 2 containing the mass occupies the same proportion of space in 3 as in 1. entropy is unchanged

Figure 14. Entropy is unchanged. 


\section{Hypotheses and Conclusion}

What can be said is that, in total, the topological modification would allow the vision of a cosmos that seems to us more coherent with verifiable theoretical consequences:

1) This cosmos respects several principles:

- First, the principle of simplicity (lex parcimoniae or principle of the Occam razor)

- This space, like others, respects the principle of Mach. The observation that there is always an apparent movement implies that there is no centre or particular point (spherical space).

2) This topology meets the theoretical prerequisites of Perelman-Poincaré which requires any space with 3 dimensions of curvature to be multiconnex. And to those of Penrose by removing the problem of the 2 infinite: the infinity of the origin point of the big bang since the central zone does not belong to space; the outer infinity since space is finite. The cosmos is then representable without the mathematical impossibilities or singularities related to the infinite.

The notion of big or small does not make sense if we do not have Cartesian benchmarks outside the system. Only the measurement of the radius of curvature can tell whether this space is with a small or large expansion. The radius of curvature may be the only possible objective measure within this space because it is not dependent on a measurement standard external to the system.

3) The movement in this space is continuous. There is no discontinuity as in the hyperspheres of Poincaré or Luminet where, we come out one side and we come back another side. (Problem of mapping a Round Earth)

4) The homogeneity of the cosmos can be explained by the shape of this space where seemingly distant points are, in fact, close to each other. This could, at least for this aspect, makes unnecessary the hypothesis of the inflation.

5) This topology induces verifiable observations:

a) In 2 opposite directions, images of the same galaxy

b) Statistically peaks of galaxies of nearby age of $7 \times 10^{9}$ years. (This proposal seems to be verified: a peak of galaxies around $2200 \mathrm{Mpc}$ seems to exist and allows to date the age of the universe around 14 billion years. However, a verification with a larger statistical series is essential).

c) The NASA/IPAC study of galaxy distances also suggests the verifiable hypothesis that if light travels around in 14 billion years, light capture may sometimes reach us after a second turn. This would then lead to the illusion of seeing galaxies that are more than 14 billion years old and all the more evolved because they are ancient.

d) Galaxies around $7 \times 10^{9}$ years are more similar

e) Galaxies, after going through half the circle tend to converge to a point.

6) The problem of the value of the "critical" mass could disappear and become irrelevant. If there is a centrifugal force, possible because of the 4th dimension of length without contradicting the rule of the geodesic, this force then has effect in 
the expansion, or even explains it (To avoid talking about force and remain in the principle of curvature of the GR, it would probably be better to start from entropy). The expansion is not infinite but due to the centrifugal force which, at a given moment, becomes equal to the gravitational force. A balance is made and then fixes a equilibrium density which is equal to what is called the critical density. Overall, in this hypothesis, the rate of expansion decreases with time and will have to be distinguished from the increase in the speed due to gravitation.

7) The force of attraction is always attractive. The shape of this space explains that a repulsive gravitation is only an optical illusion. If in the cosmos, the opposite point is at $7 \times 10^{9} \mathrm{LY}$, as soon as half of this path has been covered, despite the expansion, the masses get closer, then gravity causes a measurable acceleration, an increase in speeds and therefore an impression of acceleration of expansion (this statement also seems to be verified since an acceleration was measured at billion $6-7 \mathrm{LY}$ ). The shape of this space is well correlated with observations of the movements of Laniakea's galaxies. Black energy retains other theoretical arguments, but is no longer necessary to explain an acceleration of expansion.

8) The black holes in this space have no mathematical singularity in their center since with this topology whatever the level of contraction, there is always a zone in the center that does not belong to space.

\section{Limits of This Theory}

1) There is first the psychological problem of representing something that is counter-intuitive: the existence of a central zone that does not belong to space (it is necessary to abstain from representing an infinite Cartesian space).

2) This curved space allows to represent a spherical cosmos in 3 dimensions, but it is not said that there is no other representation of the same type (we do not respect the maximum volume given by Luminet [10]). It is not because a theory respects the principle of simplicity that it is necessarily accurate.

3) It necessarily implies since the infinite Cartesian space is virtual and not real that the cosmos is finite. It is therefore in absolute opposition to multiple spaces, "multiverse" and any infinite entity. If we postulate philosophically an infinite cosmos, then this model must be rejected.

4) It is a universe whose radius fluctuates between a maximum and a minimum and which therefore does not necessarily go to the length of Planck.

5) The description of this space does not answer the problem about the nature of finite or infinite time.

6) How to measure a radius of curvature of the order of $1^{\circ}$ for 40 million LY which could be a confirmation of this model?

Other theories offer explanations for observing very old and evolved galaxies.

This theory eliminates mathematical singularities but conjectures are still necessary for example to prevent the density from increasing without limit.

\section{Conflicts of Interest}

The author declares no conflicts of interest regarding the publication of this paper. 


\section{References}

[1] Penrose, R. (1965) Physical Review Letters, 14, 57-59. https://doi.org/10.1103/PhysRevLett.14.57

[2] Bohr, N. (1991) Physique atomique et connaissance humaine, Ed. Gallimard 2015 réed.

[3] Lamé, G. (1859) Leçons sur les coordonnées curvilignes et leurs diverse application, Mallet-Bachelier.

[4] Tegmark, M. (1998) Annals of Physics, 270, 1-51. https://doi.org/10.1006/aphy.1998.5855

[5] Gauss, C.F. (1825) CEuvres complètes, t.IV, p. 189-193.

[6] Sommerfeld, A. (1923) Atomic Structure and Spectral Lines. Londres, Methuen.

[7] Poincaré, H. 1902 () La science et l'hypothèse, chap. III; Les géométries non euclidiennes, Flammarion 2014 réed.

[8] Poincaré, H. (1895) Journal de l'école polytechnique, 1, 1-121.

[9] Poincaré, H. (1904) Rendiconti del circolo matematico di Palermo, 18, 45-110. https://doi.org/10.1007/BF03014091

[10] Luminet, J.P. (2005) L'univers chiffonné, Gallimard/folio.

[11] Luminet, J.P. (2014) La topologie cosmique p94 in Pour la science hors série $\mathrm{n}^{\circ} 83$ avril-juin.

[12] NED (NASA/IPAC Extragalactic Database)-4D: 394 Distances to 349 Galaxies with v $>1 / 8$ c Compiled from the literature by Barry F. Madore (Pasadena) and Ian P. Steer (Toronto) Updated to January 1, 2007.

[13] Tully, R.B., Courtois, H., Hoffman, Y. and Pomarède, D. (2014) Nature, 513, 71-73. https://doi.org/10.1038/nature13674

[14] Mignonat, M. (2018) Journal of Modern Physics, 9, 1545-1558. https://doi.org/10.4236/jmp.2018.98095 


\section{Appendix}

Geometric calculation of the position of any point $S$ or event in this finite $3 D$ space:

A system of curvilinear coordinates that seems relatively simple and allows us to remain in a finite space (and therefore in physics) is to consider that:

Every $S$ point in space is marked by three curvilinear coordinates $x, y, z$.

Each curvilinear coordinate $x, y, z$ is located on an arc of circle having the same radius of curvature.

A first 2D sphere is defined by the marks $i$ and $j . O$ is a point taken as origin located on this sphere. A first point $W$ is defined by its coordinates $x$ and $y . x$ and $y$ are perpendicular and located on the surface of the sphere. The mark $k$ and so the coordinate $z$ start perpendicular from the point $W$ to the surface of this first sphere.

Any point $S$ of the space can then be defined by a curvilinear coordinate $Z$ between $-\Pi / 4$ and $3 \Pi / 4$ rd (Figure 14 left) and a curvilinear coordinate $\mathrm{w}$ resulting from the curvilinear coordinates $x$ and $y$, the coordinate w corresponds to the intermediate point $W$ located on the sphere. By convention $W S$ and $O W$ are located in the same plane and always by convention the curvature for WS is done in the direction dextrogyre when $z>0$ and in the direction lévogyre when $Z<0$. (The purpose of these conventions is to define a point $S$ by a single group of coordinates and to simplify the calculations. A mathematical generalization would probably be feasible but would not add anything more to the physical representation.)

Starting from $O W$ and applying the first Gauss formula of spherical trigonometry

$(\cos w=\cos x \cdot \cos y+\sin x \cdot \sin y \cdot \cos A)$ we find, since $O x$ perpendicular to $O y<=>A=90^{\circ}$, that: $\cos w=\cos x \cdot \cos y$
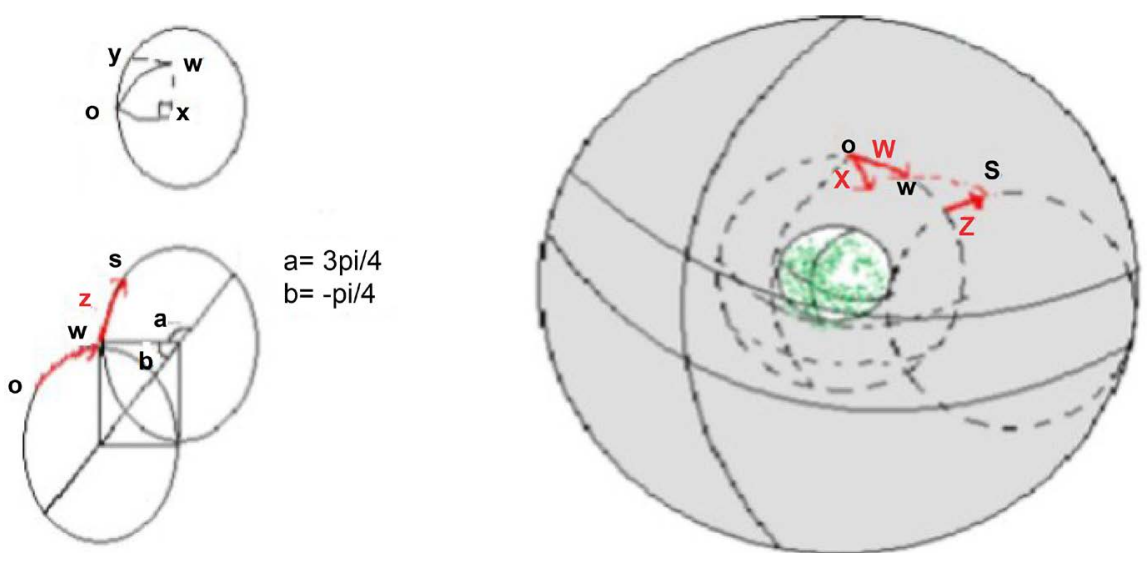

We first do the calculation for $z$ with $0<z<+3 \Pi / 4$

The coordinates $w$ and $z$ are in the same plane and the radius of curvature $R$ is the same for the 2 arcs of circle between $O W$ and $W S$ (drawing above); the radius of curvature $R^{\prime}$ of $O S$ is different and will be variable according to the 
considered point; its calculation is not necessary to position the point $S$ but interesting to do since it gives the curvature of the geodesic which becomes different according to the distance traveled. The angles $w, x, y, z$ are in radians. The lengths of the strings of the circle arcs are:

$$
O W=2 R \sin w / 2
$$

$$
\begin{gathered}
W S=2 R \sin z / 2 \operatorname{arc} W S \perp \operatorname{arc} W O, \text { so ang. } O W S=w / 2+\Pi / 2+z / 2 \\
O S=2 R^{\prime} \sin s / 2
\end{gathered}
$$
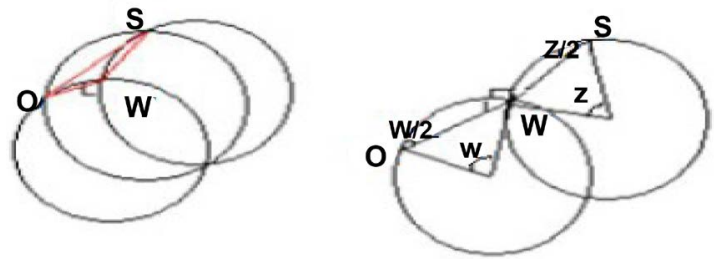

We apply the theorem of Al-Kashi:

$$
\begin{gathered}
O S^{2}=O W^{2}+W S^{2}-2 O W \cdot W S \cdot \cos (w / 2+z / 2+\Pi / 2) \\
O S^{2}=4 R^{2}\left[\sin ^{2} w / 2+\sin ^{2} z / 2-2 \sin w / 2 \sin z / 2 \cos (w / 2+z / 2+p i / 2)\right] \\
O S^{2}=4 R^{2}\left[\sin ^{2} w / 2+\sin ^{2} z / 2+2 \sin w / 2 \sin z / 2 \sin (w / 2+z / 2)\right] \\
O S^{2}=4 R^{2}\left[\sin ^{2} w / 2+\sin ^{2} z / 2+\sin ^{2} w / 2 \sin z+\sin w \sin ^{2} z / 2\right] \\
O S^{2}=4 R^{2}\left[(1+\sin z) \sin ^{2} w / 2+(1+\sin w) \sin ^{2} z / 2\right]
\end{gathered}
$$

This formula gives the distance of the $O S$ straight segment that can be used in the geometric representation when this finite space is immersed in the infinite Cartesian space that we consider virtual. We keep $R$ which, as part of the expansion of the universe can be replaced by $R=R_{0}(t)$

( $c=$ speed of light, $t=$ time) We could then write for the coordinates of an event:

$$
O S^{2}=c^{2} t^{2}-4 R_{0}^{2}(t)\left[(1+\sin z) \sin ^{2} w / 2+(1+\sin w) \sin ^{2} z / 2\right]
$$

If we want to determine the curvature of the geodesic connecting $O$ and $S$, we can also write:

$$
4 R^{\prime 2} \sin ^{2} s / 2=4 R^{2}\left[(1+\sin z) \sin ^{2} w / 2+(1+\sin w) \sin ^{2} z / 2\right]
$$

so:

$$
R^{\prime 2}=R^{2}\left[(1+\sin z) \sin ^{2} w / 2+(1+\sin w) \sin ^{2} z / 2\right] / \sin ^{2} s / 2
$$


Case where $-\Pi / 4<z<0$

Then the angle $O W S=z / 2+\Pi / 2-w / 2$ so,

$$
\begin{gathered}
O S^{2}=4 R^{2}\left[\sin ^{2} w / 2+\sin ^{2} z / 2+2 \sin w / 2 \sin z / 2 \sin (-w / 2+z / 2)\right] \\
O S^{2}=4 R^{2}\left[\sin ^{2} w / 2+\sin ^{2} z / 2-\sin ^{2} w / 2 \sin z+\sin w \sin ^{2} z / 2\right] \\
O S^{2}=4 R^{2}\left[(1-\sin z) \sin ^{2} w / 2+(1+\sin w) \sin ^{2} z / 2\right]
\end{gathered}
$$

The formula giving the right segment $O S$ as a function of time becomes:

$$
O S^{2}=c^{2} t^{2}-4 R_{0}^{2}(t)\left[(1-\sin z) \sin ^{2} w / 2+(1+\sin w) \sin ^{2} z / 2\right]
$$

The formula (7) becomes:

$$
R^{\prime 2}=R^{2}\left[(1-\sin z) \sin ^{2} w / 2+(1+\sin w) \sin ^{2} z / 2\right] / \sin ^{2} s / 2
$$

\section{In total:}

Each point of this finished and representative space, multiconnex, with a spherical type and constant curvature and 3 dimensions, can be located by 3 coordinates curvilinear $x, y, z$.

Knowing that $\cos w=\cos x \cdot \cos y$, then,

When: $0<z<+3 \Pi / 4$ :

$$
\begin{gathered}
\sin ^{2} s / 2=\left(R / R^{\prime}\right)^{2}\left[(1+\sin z) \sin ^{2} w / 2+(1+\sin w) \sin ^{2} z / 2\right] \text { or } \\
O S^{2}=4 R^{2}\left[(1+\sin z) \sin ^{2} w / 2+(1+\sin w) \sin ^{2} z / 2\right]
\end{gathered}
$$

When: $-\Pi / 4<z<0$ :

$$
\begin{gathered}
\sin ^{2} s / 2=\left(R / R^{\prime}\right)^{2}\left[(1-\sin z) \sin ^{2} w / 2+(1+\sin w) \sin ^{2} z / 2\right] \text { or } \\
O S^{2}=4 R^{2}\left[(1-\sin z) \sin ^{2} w / 2+(1+\sin w) \sin ^{2} z / 2\right]
\end{gathered}
$$

If we take a temporal dimension by placing the radius $R=R_{0}(t)$, we create a space of Minkowski type but which is finite and representative.

An event can be located by the segment $O S$ such as:

$$
O S^{2}=c^{2} t^{2}-4 R_{0}^{2}(t)\left[(1+\sin z) \sin ^{2} w / 2+(1+\sin w) \sin ^{2} z / 2\right]
$$

when $0<z<+3 \Pi / 4$

$$
O S^{2}=c^{2} t^{2}-4 R_{0}^{2}(t)\left[(1-\sin z) \sin ^{2} w / 2+(1+\sin w) \sin ^{2} z / 2\right]
$$

when $-\Pi / 4<z<0$ 